\title{
Structural, Optical and Electrical Analysis of Pentacene Thin Film
}

\author{
S. Sindhu, K. Shree Krishna Kumar
}

\begin{abstract}
The significant electrical characteristics of pentacene thin film was studied by fabricating silver-pentacene-aluminium sand witch device. This metal-semiconductor-metal (MSM) device can act as the diode. When investigating, it is found that the diode can act as an equivalent plate capacitor as reverse bias mode exclusive of current flow. In forward bias mode, studied diode as an equivalent circuit with a parallel circuit of resistance -capacitance. This paper describes analysis of capacitance by measuring I-V characteristics and impedance measurement. The free samples of pentacene are also analysed using field emission scanning electron microscope (FESEM) and X-ray diffraction micrographs $(X R D)$. The X-ray diffraction micrograph shows the configuration of pentacene in the (-1 02 2) plane as the strongest orientation. The particle size are analysed using FESEM. The optical characteristics are analysed using $U V$ spectrometer. The spectra of absorption, reflection and refraction are studied well.
\end{abstract}

Keywords: XRD, FESEM, UV, Pentacene, optical spectra, capacitance

\section{INTRODUCTION}

Organic semiconductors are now a days commonly used for the fabrication of thin film devices. They show high potential ,low expense, light weight and gradually increasing performance[1]. They can be used as active layers for different types of thin film transistors. It can also be used as optoelectronic devices and in displays. They can be used in programmable devices, high frequency diodes and memories

In this study we used pentacene an aromatic hydrocarbon and its morphological and structural characteristics are analyzed first. It is a very promising material and this lead to the study of the optical properties. The electrical and optical chara are comparable. We analyzed the samples using field emission scanning electron microscopy (FESEM), and X-Ray diffraction (XRD) analysis. The optical properties are analyzed using UV spectrometer. The absorption spectrum is studied using absorption spectroscopy by measuring the absorption of light as a function of frequency and wavelength .The reflection and refraction spectra are also analyzed to study the optical properties. Organic materials can be fabricated as active layers for different thin film transistors. In this work the electrical characteristics of two different thickness thin films are compared. Pentacene thin film can be used for the fabrication of solar cells.

\section{Revised Manuscript Received on December 15, 2019}

*S. Sindhu, School of Technology and Applied Science, Mahatma Gandhi University, Kottayam, Kerala, India.

E-mail: sindhumanojvard@gmail.com

Dr. K. Shree Krishna Kumar, School of Technology and Applied Science, Mahatma Gandhi University, Kottayam, Kerala, India.

E-mail:kshreekk@gmail.com

\section{EXPERIMENT}

Pentacene thin films are coated on the clean glass substrate using physical vapour deposition technique. It is the best coating method for organic materials. Before coating the glass substrate is ultra sonically cleaned .It is sonicated using methanol and acetone for 5 minutes each .It is rinsed systematically using distilled water and dried for $2 \mathrm{hrs}$ at 6600 C. The glass slide and the organic material pentacene are placed in the vacuum chamber. The distance between the glass substrate is less than the mean free path of the material.

Pentacene coated with different thickness on a glass substrate and studied two samples with thickness $300 \mathrm{~nm}$ and $400 \mathrm{~nm}$. We investigated the thin film by field emission scanning electron microscope (FESEM) . SEM analysis is done to study the surface analysis. Using FESEM we studied the size and shape of the particles.

Next we analysed the thin film using X-Ray diffraction image. The XRD pattern is shown below. We also studied the optical properties using UV/V spectrometer and analyzed the absorption , reflection and refraction of the light falling on it. The electrical characteristics are studied using silver and aluminium as electrodes on both sides. The thickness of the electrodes are 400nm and the active are is $10 \times 10 \mathrm{~mm} 2$. The VI characteristic are measured using Keethly sourcemeter. The thin film of $300 \mathrm{~nm}$ is fabricated with area $10 \times 10 \mathrm{~mm}$. The process is repeated with $400 \mathrm{~nm}$ thickness thin film. The process conditions are kept same for both films in the same evaporation chamber. The vacuum evaporation is done under the pressure of $2 \times 10-5$ mbar with keeping substrate at room temperature $240 \mathrm{C}$. The pentacene evaporation rate is kept as $.1 \mathrm{~nm} / \mathrm{s}$. The current and voltage to get V-I characteristics were measured using source meter (Keithley4200). The impedance is measured using impedance analyser HP 4192A LF. The organic semiconductor layer thickness is exactly determined using profilometer, Dektak 150 surface profiler. The electrical measurements at room temperature were calculated.

\section{RESULTS AND ANALYSIS}

a. FESEM Analysis: Field Emission Scanning Electron Microscopy (FESEM) provides topographical and elemental information at magnifications of $10 x$ to $300000 x$ with virtually unlimited depth of field. 


\section{Structural, Optical and Electrical Analysis of Pentacene Thin Film}

FESEM images of pentacene thin films with different magnifications are shown below in fig.1. The morphology of the surface of thin film can be studied using FESEM. Field Emission SEM (FESEM) produces clear , less electrostatically distorted images with spatial resolution down to $11 / 2$ nanometers -three to six times better

It is of uniform distribution of almost round shaped particles . It is observed that the average size of the particle is $70-90 \mathrm{~nm}$ range.

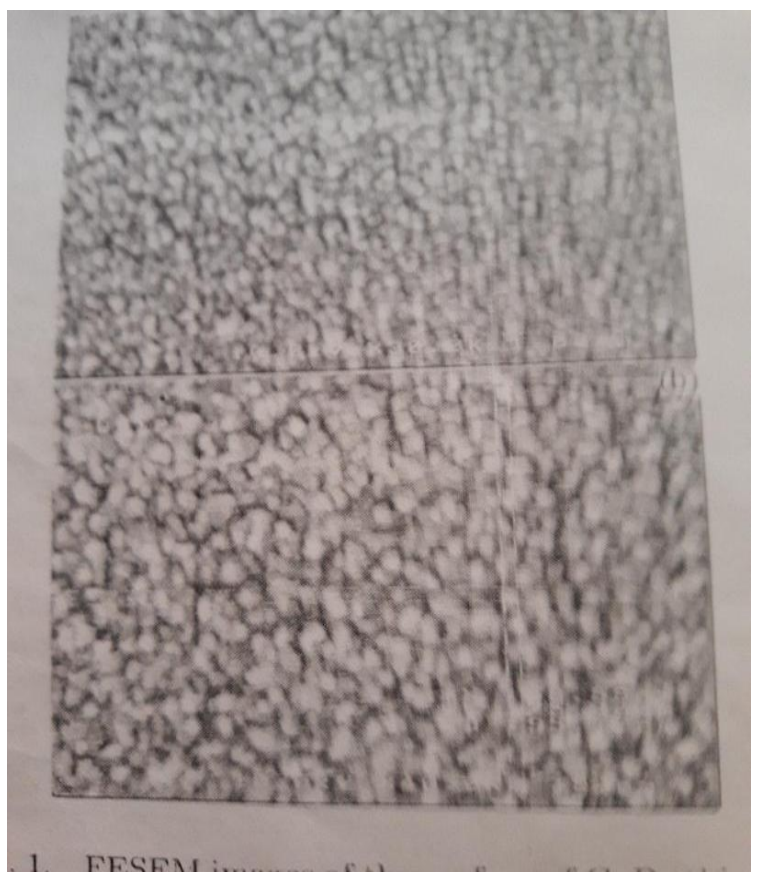

Fig.1FESEM images of the surface of pentacene thin film at (a) $750 \mathrm{~nm}$ and (b) $500 \mathrm{~nm}$ magnifications indicating the mean particle diameter to be $70-90 \mathrm{~nm}$

b. X-Ray Diffraction Analysis : The X-ray diffraction pattern of the pentacene thin film is shown in figure.2. The XRD microscope indicates the configuration of alpha form with the plane (-102)) as the preferred orientation.[2] Even though more than one peaks available in the pattern the angle $2 \theta$ of 19.067 and hkl values of (-102) is the strongest one and can be selected. The -Ray diffraction data for pentacene is shown in the table. The mean crystalline size can be calculated by Scherrer's formula

$$
\mathrm{L}=\mathrm{ks} \lambda / \beta 0 \operatorname{Cos} \theta \ldots \ldots(\text { Eq. } 1)
$$

Where $\lambda$ is the $\mathrm{x}$-ray wave length $\left(1.540 \mathrm{~A}^{0}\right), \beta 0$ is the full width at half maximum (FWHM) of the most intense peak in radiations, $\theta$ is the Bragg's angle and ks is the Scherrer's constant $=0.9$.

$\alpha=76.62, \beta=88.74, \gamma=63.85, \mathrm{z}=2$.

Molecular weight $=278.35$, volume $(C D)=637.78$, $\mathrm{Dx}=1.449$

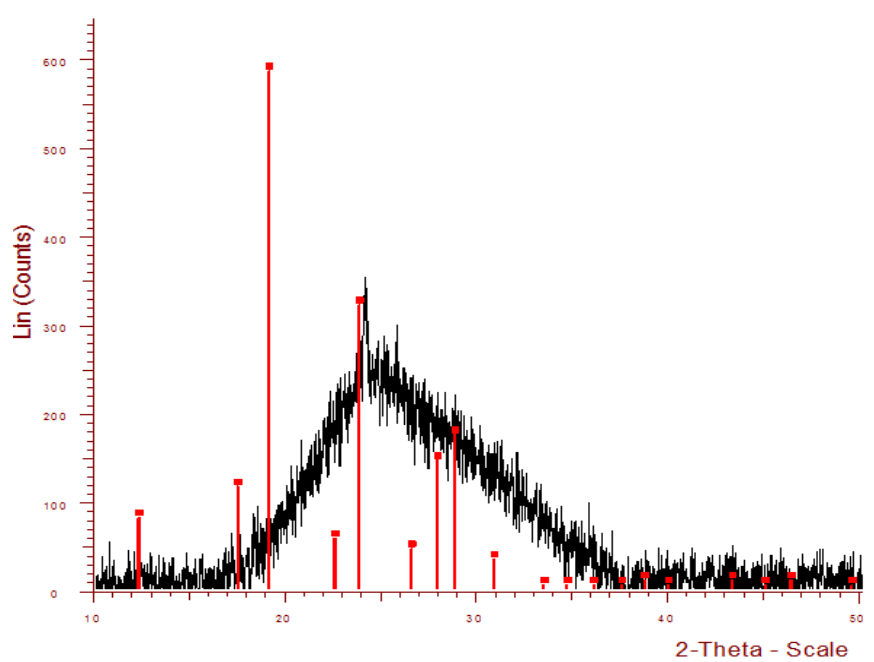

Fig. 2. X-ray diffraction micrograph of pentacene thin film with the thickness of $400 \mathrm{~nm}$.

\begin{tabular}{rrr|r|r|l}
\multicolumn{1}{l|}{ 2th } & \multicolumn{1}{l|}{ l } & \multicolumn{1}{l}{$\mathrm{h}$} & $\mathrm{k}$ & $\mathrm{l}$ & Comments \\
\hline 6.003 & 65 & 0 & 0 & 1 & \\
12.232 & 14 & 0 & 0 & 2 & \\
17.443 & 20 & 1 & 0 & 1 & \\
19.067 & 100 & -1 & 0 & 2 & Strongest line. \\
22.526 & 10 & 1 & 1 & 3 & \\
23.810 & 55 & 1 & 2 & 0 \\
26.539 & 8 & 1 & -1 & 1 \\
27.902 & 25 & -1 & 1 & 3 \\
28.823 & 30 & 2 & 1 & 1 \\
30.862 & 6 & 2 & 2 & 0 \\
33.498 & 1 & -1 & 0 & 5 \\
34.715 & 1 & -2 & -2 & 2 \\
36.101 & 1 & 1 & 3 & 3 \\
37.588 & 1 & 2 & 1 & 4 \\
38.766 & 2 & 1 & 1 & 6 \\
40.022 & 1 & 1 & 2 & 6 \\
43.385 & 2 & 3 & 2 & 1 \\
45.092 & 1 & 2 & 3 & 5 \\
46.459 & 2 & 3 & 2 & 3 \\
49.642 & 1 & -2 & 2 & 1 \\
51.161 & 1 & 1 & 1 & 8 \\
52.166 & 1 & 2 & -2 & 1 \\
56.178 & 1 & 0 & -4 & 1 \\
59.179 & 1 & -1 & 3 & 7 \\
61.936 & 1 & -2 & 3 & 3 \\
67.583 & 1 & -1 & 2 & 10 \\
69.348 & 1 & 0 & 5 & 0 \\
& & & &
\end{tabular}

c. UV Analysis : Ultraviolet spectroscopy is used for the characterization of absorption, reflection and refraction of organic thin films coated on plane glass[3]. The spectroscopy is a technique of measurement of light absorbed by the sample for given wavelength of light. The wavelength range here used is $300 \mathrm{~nm}$ to $800 \mathrm{~nm}$ at room temperature. 
(i)Absorption: Absorption spectroscopy is a technique used to measure the absorption of light as a function of wavelength and frequency from the radiating field $\mathrm{N}$ type $\pi$ electrons or nonbonding electrons absorb visible or ultraviolet light energy and excite the electrons to the higher antibonding molecular orbits. The electrons jump from HOMO to LUMO can absorb light with longer wavelength and minimum energy needed to jump into vibrational level. The possible type of transitions is $\left(\pi-\pi^{*}, \mathrm{n}-\pi^{*}, \sigma-\sigma^{*}\right.$, and $\left.\mathrm{n}-\sigma^{*}\right)$ and they can be ordered as follows $\sigma-\sigma^{*}>\mathrm{n}-\sigma^{*}>\pi-\pi *>\mathrm{n}-\pi^{*}$. The fig.3 shows the variation of absorption of uv radiation with wavelength. The absorption is maximum at around $500 \mathrm{~m}$ and reaches to zero after $700 \mathrm{~nm}$. Fig. 4 explains the normalized uv-v graph of powder and solution type materials. The change in absorption is due to the change in the property of the medium when adding water.

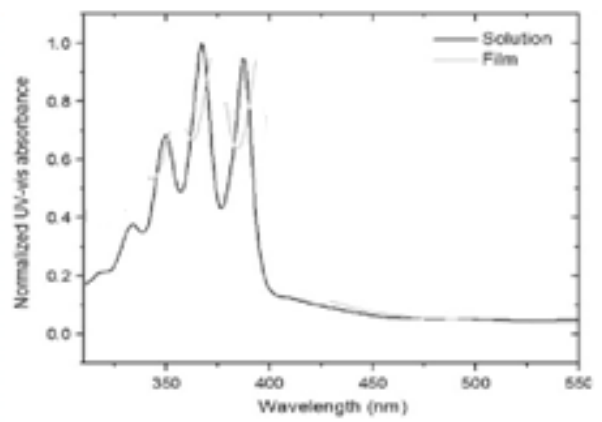

Fig.4 Normalised UV-V graph of pentacene thin film of $400 \mathrm{~nm}$ thickness.

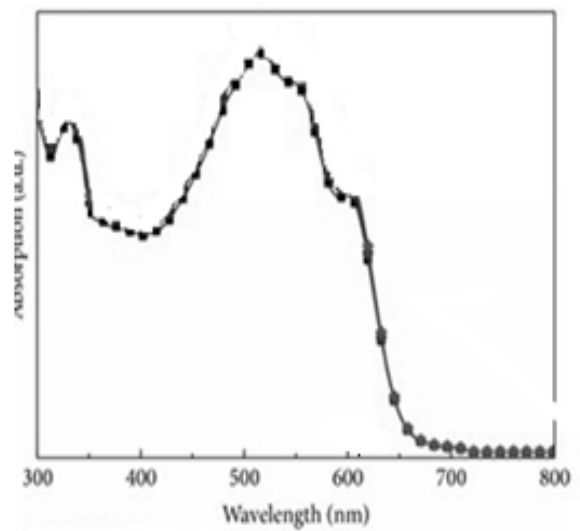

Fig.3 Absorption spectrum of pentacene thin film of $400 \mathrm{~nm}$ thickness

(ii)Reflection: Spectrum of rough samples and powders can be analyzed by using this type of diffused reflection technology. Diffuse reflection is the reflection of a wave from a surface such that it is scattered at many angles instead of calculating only one reflected ray on the surface. In the figure.5 the reflectivity slightly increases and reaches maximum above $700 \mathrm{~nm}$.

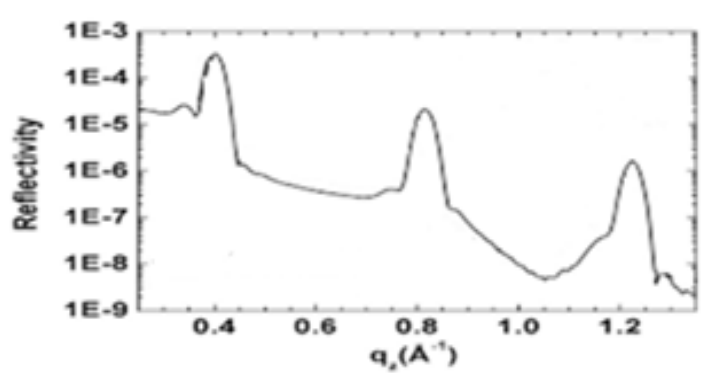

Fig.6 Reflectivity graph

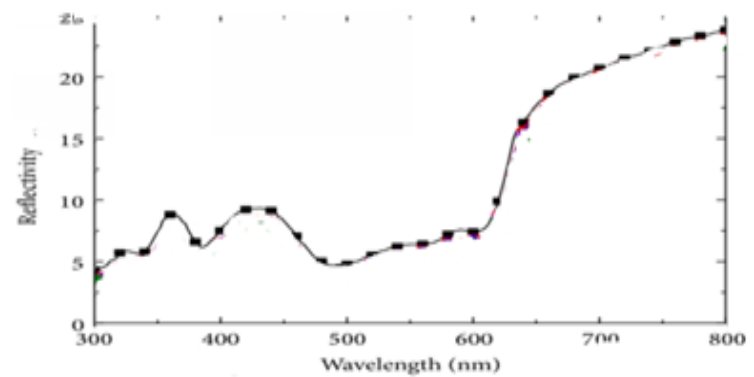

Fig. 5 Reflective spectrum of pentacene thin film with thickness $400 \mathrm{~nm}$

(iii)Refraction: Refraction is the change in direction of the light when the medium of transmission is changed. Refractive index is the ratio of speed of light in vacuum to that in the second medium. Absorption coefficient is the measure of the attenuation caused by absorption of energy per distance that occurs in the uv wave of particular wavelength propagating through a material medium of given refractive index. The value of absorption index.

$\mathrm{K} '=\mathrm{k} \lambda / 4 \pi \mathrm{n} \ldots \ldots($ Eq. 2$)$

where $\mathrm{K}$ is the absorption coefficient, is the wavelength in vacuum, and $\mathrm{n}$ is the refractive index of the absorptive material medium.

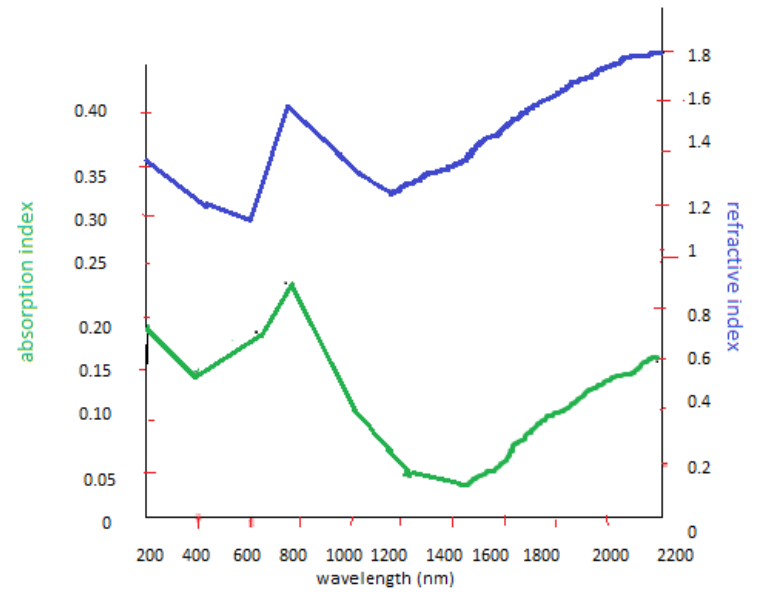

Fig.7 Variation of refractive index and absorption index. 


\section{Structural, Optical and Electrical Analysis of Pentacene Thin Film}

d. current-voltage analysis: The fig.8 shows V-I characteristics of the pentacene MSM diode with two different thickness of $300 \mathrm{~nm}$ and $400 \mathrm{~nm}$ [4]. The active area is $10 \times 10 \mathrm{~mm} 2$. The applied voltage $\mathrm{Va}$ is connected between two electrodes such as silver is the anode and aluminium is the cathode which is grounded. The current measured is very low at reverse bias mode $(\mathrm{Va}<0)$ owing to low leakage current from Al. The current increases suddenly in forward biased because of the holes injected from the side of Ag electrode. The rectification ratio is around $106(400 \mathrm{~nm})$ and 104 $(300 \mathrm{~nm})$ respectively for both samples and it is comparable with organic diodes.

Due to the very low carrier density of organic semiconductors, impedance-voltage measurements were taken to prove the full depletion in organic semiconductors. So we can prove that there is no partial depletion when $\mathrm{Va}=0$. It is not easily evident in very low thickness thin films. The schottky nature is found based on the depletion region formation and the fully depleted organic layer is due to the pure organic semiconductors low carrier density. The organic semiconductor is fully depleted and shows voltage independency when reverse biased.

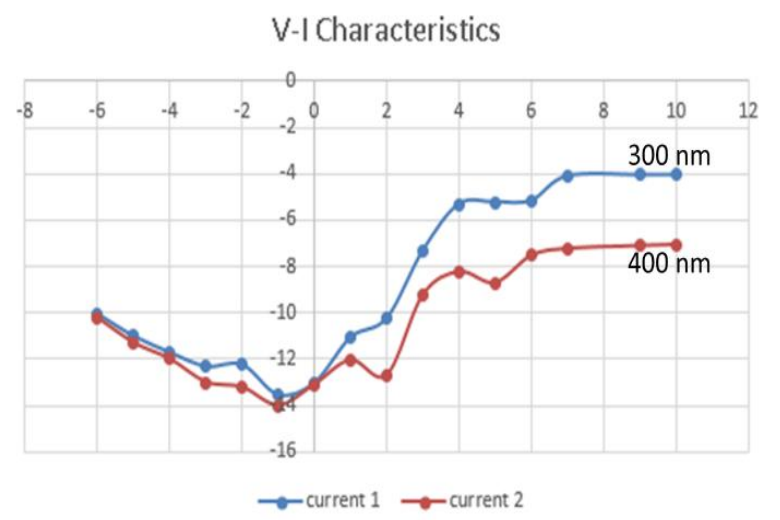

\section{Fig.8 V-I characteristics of thin film with $300 \mathrm{~nm}$ and $400 \mathrm{~nm}$ thickness.}

e. impedance analysis : The forward and reverse biased regions are evidently noticeable in fig.9. In the reverse biased region $(\mathrm{va}<0)$ the phase angle is always $-90 \mathrm{o}$ and it indicates the capacitance behavior and it is very clear that pentacene layer is completely depleted. This full depletion is due to the low doping density of the organic semiconductors. The OLEDs also shows this full depletion behavior which have thin layers with thickness in nano range. In the forward biased condition ,the diode is not capacitive because phase angle approaches 00. But it can be derived from the formula

$\mathrm{Z}=1 / \mathrm{j} \quad \mathrm{C} \ldots \ldots . .(\mathrm{Eq} 3)$

Where $\omega$ is the angular frequency of the applied voltage and C is the capacitance. $\mathrm{Z}$ is the ratio of phasor current and phasor voltage..Since $\mathrm{j}$ is an imaginary number, $\mathrm{Z}$ is a complex and its modulus and phase angle can be analyzed separately. The impedance modulus is high when reverse biased with high applied frequency. The modulus decreases suddenly when the ac frequency is below $80 \mathrm{khz}$.
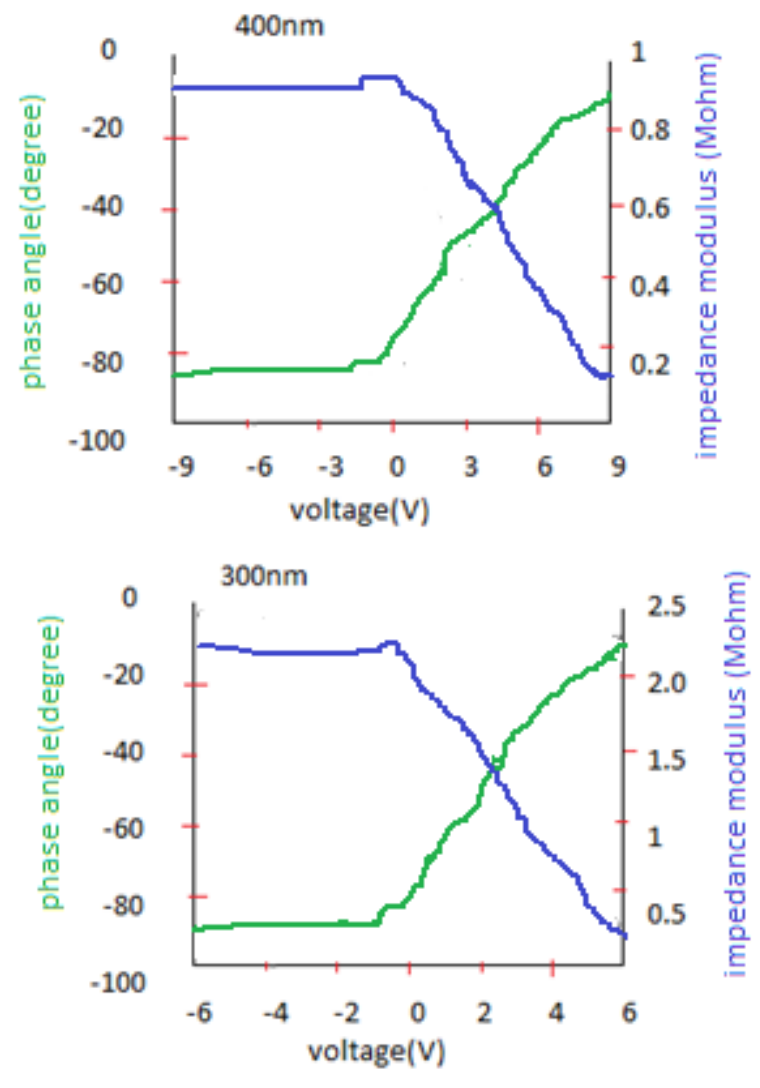

Fig.9 Impedance-voltage (Z-V) show fully depleted organic layer in the reverse-bias mode with thickness $300 \mathrm{~nm}$ and $400 \mathrm{~nm}$

f. metal-insulator-metal model : Fig.10 shows energy level diagrams and it is drawn in terms of work function of the electrode metals and pentacene[18]. The work function of aluminium is $4.7 \mathrm{eV}$ and that of silver is $4.2 \mathrm{eV}$. The ionization potential and electron affinity is $5.2 \mathrm{eV}$ and $2.8 \mathrm{eV}$ respectively. $\mathrm{Vd}$ is the built in potential which is the difference between work functions of two electrodes and it is $0.5 \mathrm{eV}$ here .Injection of holes from $\mathrm{Al}$ to $\mathrm{HOMO}$ level of pentacene is negligible due to high injection barrier of $1 \mathrm{eV}$ But the injection barrier is low, $0.3 \mathrm{eV}$ from Ag to HOMO level of pentacene and can supply high density of injected holes. So Ag can be called injection diode and Al can be called blocking diode injection diode and $\mathrm{Al}$ can be called blocking diode.

In the reverse biased condition due to very low carrier injection from two side electrodes, there is no current flow. The diode can act as a perfect capacitor.

When the diode is forward biased the conduction is in two modes. When the forward biasing voltage is lower than $\mathrm{Vd}$, the injection holes produces current. When the biasing voltage is higher than $\mathrm{Vd}$ the injection holes are not significant and charges crossing the semi-conductor layer determines current. 


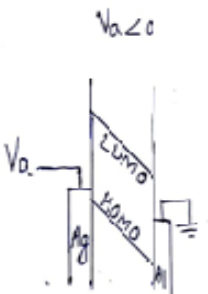

(a)

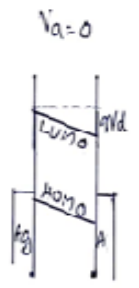

(b)

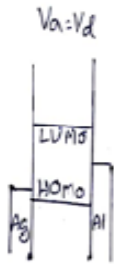

(c)

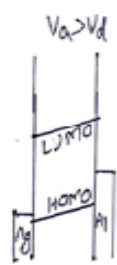

(d)

Fig 10 MIM type energy diagram of pentacene diode at different operation modes. (a) reverse bias (b) thermal equilibrium (c) flat band condition (d) forward bias.

\section{CONCLUSION}

FESEM images and XRD micro graphs where studied to investigate the morphology of the samples. The XRD micrograph indicates the configuration of pentacene with $(-102)$ plane as preferred orientation. FESEM images shows the shape and size of the particles. The optical properties like absorption, reflection and refraction of pentacene thin film according to the variation of wavelength of UV radiation is studied. The absorption and refractive indices where studied with respect to different wavelengths. The capacitive property and V-I characteristics of metal insulator metal sandwich model was analyzed.

\section{REFERENCES}

1. E.M. El-Menyawy, H.M. Zeyada, M.M. El-Nahass, Solid State Sci. 12, 2182 (2010)

2. Hsieh-ChengHan,Chi-Ang T seng, J. Mater. Chem,2012,22,22899

3. M.E.Azim.Araghi,S.Haji Mirza Mohammadi,Acta Physica Polonica A, Vol.125(2014)1

4. Dipok Saikia and P.K. Saikia,ECS Solid State Letters, 4 (11) Q51-Q54(2015).

5. Jiang.S.X.,Qin.,Guo,R.H.Zhang,L.Surface Coating and Technology 204(2010)3662

6. Chopra K.L, Thin Film Phnomena,Robert E.kreiger Publishing Company,New York, 1979

7. Harsha, K.S $>$ S.,Principle of vapour deposition of thin films First Edition,Elsevier Ltd,Oxford,U.K

8. Helmerson, u.,Latteman.M.,Bohlmark,J.,Ehiasarian, A.P.,Gudmundsson,J.T.,Thin Solid Films,513(2006)1.

9. Hong,Y.K.,Lee,C.Y.,Jeing.,C.K.,Sim,J.H.,Kim,K., Joo J.,Kim,M.S.Lee,J.Y.,Jeong,S.H.,Byun,S.W.,Current Applied Physics, 1(2001) 439.

10. Ryssel.H.,Glawischnig, H., Implantation Techniques,Springer-Verlag,Berlin,1982.

11. Ning Zhang,Jianchun Jiang,Xiangyu Li, Yajuan Tong, Radiation Physics and Chemistry,80 (2011) 990

12. Wong,K.K.H.,Zinke-Allmang., Wan, W.K.Nuclear Instruments and Methods in Physics Research Section B: Beam Interactions with Materials and Atoms,249(2006)362

13. L. Holland, Vacuum deposition of thin films, Chapman and Hall,London (1956)

14. D.M.Mattox. Thin Solid Films 124,3 (1985)

15. L.I.Maissel and R.Glang, Hand book of thin film Technology ,McGraw

16. R.Roth.Vacuum Technology, North Holland, Amsterdam(1976)

17. H.C.Cash well,Physics of thin films,vol.1.Ed.G.Hass Academic Press,New York(1963)

18. B.Jaeckel, J.B.Sambur and B.A. Parkinson, Applied Physics, 103 063719(2008 